\title{
CORRELATION OF SERUM ZINC LEVELS WITH SEVERITY OF ACNE VULGARIS
}

\author{
Gunda Praneeth Kumar1, Tina Priscilla²
}

${ }_{1}^{1}$ Assistant Professor, Department of DVL, Apollo Institute of Medical Sciences and Research.

${ }^{2}$ Assistant Professor, Department of DVL, Apollo Institute of Medical Sciences and Research.

\section{BACKGROUND}

ABSTRACT

Acne vulgaris is a chronic inflammatory disorder of the pilosebaceous duct characterised by polymorphic lesions of comedones, papules, pustules, nodules and cysts. It occurs most commonly in adolescents and young adults. Zinc has been used extensively both topically and systemically for the management of acne vulgaris. Zinc levels in serum and skin has received the attention of many researchers in the field.

\section{MATERIALS AND METHODS}

A prospective observational study was conducted in patients presenting with Acne vulgaris to the dermatology outpatient clinic of KLE Hospital in Belgaum, Karnataka between June 2011 and June 2012. A total of 70 patients whoever has given consent and satisfying inclusion criteria were included in the study. Only patients who had not taken any zinc therapy were selected for the study. Fasting venous blood was collected from each patient. The serum zinc levels of all the samples were estimated by using immunoturbidometry method. Patients were divided into two groups: Group 1: Acne vulgaris grade 1 and 2 (mild-to-moderate acne). Group 2: Acne vulgaris grade 3 and 4 (severe acne). The mean serum level of zinc in relation to severity of the disease was calculated using t-test analysis.

\section{RESULTS}

There was no correlation between serum zinc level and severity of acne and the results were statistically not significant $(P$ value $>$ 0.05).

\section{CONCLUSION}

There is no correlation between serum zinc levels and severity of Acne vulgaris.

\section{KEYWORDS}

Acne Vulgaris, Zinc.

HOW TO CITE THIS ARTICLE: Kumar GP, Priscilla T. Correlation of serum zinc levels with severity of acne vulgaris. J. Evolution Med. Dent. Sci. 2018;7(05):560-563, DOI: 10.14260/jemds/2018/127

\section{BACKGROUND}

Acne vulgaris is a common chronic skin disease involving blockage and inflammation of pilosebaceous units (hair follicles and their accompanying Sebaceous gland). Acne can present as non-inflammatory lesions, inflammatory lesions or a mixture of both affecting mostly the face, but also the back and chest. In fact, no individual can cross adolescence without a few comedones and papules. It has lot of psychological impact on teenage girls. Sulzberger and Zaidems in their article stated that- "There is no single disease that can cause more psychological trauma, more maladjustment between parent and children and feelings of inferiority than does acne." ${ }^{1}$

There are many myths about acne like its infectivity and relation to chocolate etc. It is unfortunate that aetiology of such a common condition still has many controversies and is unclear. Recently, it has been shown that consumption of foods with high glycaemic index has led to aggravation

'Financial or Other Competing Interest': None.

Submission 02-07-2017, Peer Review 13-01-2018,

Acceptance 18-01-2018, Published 29-01-2018.

Corresponding Author:

Dr. Tina Priscilla

Flat No. 403, Super Corona Apartments,

Near Food Bazaar, Ou Colony Main Road,

Shaikpet, Hyderabad-500008.

E-mail: drtina_priscilla@apolloimsr.edu.in dr_tina_k@yahoo.com

DOI: $10.14260 /$ jemds $/ 2018 / 127$

\section{(c) (1) $(9)$}

of acne. Zinc, elemental or in its various forms (salts), has been used as a therapeutic modality for centuries. The role of trace elements like copper, iron, magnesium etc., have also been studied, but there is no consensus among the researchers as to their significance in acne. The serum zinc levels in acne in various studies contradict each other. Many of the workers have concluded that there is no change in serum zinc levels and acne, whereas others have found a remarkable decrease in the levels of zinc.

\section{Objective}

We aimed to evaluate the serum zinc level in patients with acne vulgaris and correlate with the severity of disease.

\section{MATERIALS AND METHODS}

This study was approved by the Ethics Committee of KLE Hospital, Belgaum, Karnataka.

\section{Study Design}

Prospective observational study.

\section{Study Period}

June 2011 and June 2012

\section{Sampling Procedure}

Out of all the acne vulgaris patients attending the Dermatology outpatient clinic of KLE Hospital in Belgaum, Karnataka between June 2011 and June 2012, 70 patients whoever gave consent were recruited as the study subjects. 
Only patients who had not taken any zinc therapy were selected for the study. A detailed history was taken from each patient regarding his/ her age, sex and occupation. Patients were also asked about history of acne in the family. Each patient was examined thoroughly, and a note was made of the distribution of lesions, type of lesions and associated skin and systemic diseases.

The acne lesions in each patient were graded according to the grading method described by Tutakne and Chari.

\section{Inclusion Criteria}

a) Being over 15 years of age.

b) Being at least a primary school graduate.

c) Willing to participate in the study.

\section{Exclusion Criteria}

a) Any disfiguring facial condition other than acne.

b) History of active malignancy.

c) Under immunosuppressive treatment.

d) Liver cirrhosis.

e) Renal failure.

f) Pregnancy.

g) Alcoholism.

h) Malabsorption disorders.

i) Physical disability.

j) Neurological disorder.

Written consent was taken from all the study subjects. Fasting venous blood was collected from each patient. The blood was centrifuged at $2000 \mathrm{rpm}$ for 15 minutes and the serum was collected in a plastic container with an air tight cap, labelled. Glass containers were deliberately avoided to prevent zinc contamination from glass. The serum samples were analysed 'for zinc levels at the KLE Hospital. Serum immunoturbidometry method was the method used for estimation of serum zinc levels. This is one of the standard methods of estimation of serum zinc levels. The patients were divided into two groups,

- Group 1 include patients with Acne Grade 1 and Acne Grade 2.

- Group 2 include patients with Acne Grade 3 and Acne Grade 4.

There were 58 patients in Group A and 12 patients in Group B based on the Grades of Acne vulgaris. Serum zinc levels were estimated in both the groups. The mean serum level of zinc in relation to severity of the disease was analysed based on the t-test analysis.

\section{Statistical Analysis}

Continuous data were expressed as mean \pm SD and median and analysed by unpaired ' $\mathrm{t}$ ' test. Categorical data was represented as numbers and percentages and was analysed using the Chi-square test.

$P$ value of 0.05 or less was considered as statistically significant. Study was analysed by SPSS version 22.0.

\section{RESULTS}

Among the 70 cases that were studied, maximum cases of Acne Vulgaris were seen in the age group of $16-20$ years $(47.14 \%)$.

\begin{tabular}{|c|c|c|}
\hline Age in Years & No. of Cases & Percentage \\
\hline $11-15$ & 4 & $5.714 \%$ \\
\hline $16-20$ & 33 & $47.142 \%$ \\
\hline $21-25$ & 28 & $40 \%$ \\
\hline $26-30$ & 4 & $5.714 \%$ \\
\hline $31-35$ & 1 & $1.428 \%$ \\
\hline \multicolumn{3}{|c|}{ Table 1. Age Incidence } \\
\hline
\end{tabular}

Males had maximum prevalence at 18 years and females at 18 and as well as 19 years. The youngest patient was 12 years old and the oldest was 32 years old. Overall incidence among males was $54.71 \%$ and among females it was $45.28 \%$. Male: Female ratio in this study was 1.0588: 1. A slight male preponderance was noted. Most of the patients were students. $38.57 \%$ of the patients gave a family history of acne, either in the siblings or the parents. Thus, this agrees with the fact that hereditary predisposition is present in Acne Vulgaris, but other factors are also involved. $82.35 \%$ of female patients gave a history of premenstrual flare up of their lesions.

\begin{tabular}{|c|c|c|}
\hline Sex & Males & Females \\
\hline No. of cases & 36 & 34 \\
\hline Percentage & $51.43 \%$ & $48.57 \%$ \\
\hline \multicolumn{3}{|c|}{ Table 2. Sex Incidence } \\
\hline
\end{tabular}

Most of the cases in the present study belonged to grade II acne (57.14\%). Severe acne (grade III and grade IV) cases amounted to $15.174 \%$ and among these $91.67 \%$ were males and $8.33 \%$ were females. Thus, males had more severe acne than females.

\begin{tabular}{|c|c|c|}
\hline Grades & No. of Patients & \% \\
\hline I & 18 & 25.714 \\
\hline II & 40 & 57.142 \\
\hline III & 10 & 14.28 \\
\hline IV & 2 & 2.85 \\
\hline \multicolumn{2}{|c|}{ Table 3. Severity of Acne } \\
\hline
\end{tabular}

$55.174 \%$ of the patients gave a history of manipulating their lesions. $28.57 \%$ of the patients reported a summer exacerbation and $5.71 \%$ had winter exacerbation. None of the patients had any improvement in their lesions in summer. $40 \%$ of the patients related worsening of their lesions to fatty food, non-vegetarian food and chocolates. Though this was a subjective finding, it was quite significant in our study. $77.14 \%$ of the patients had seborrhoea as an associated feature.

\begin{tabular}{|c|c|c|c|}
\hline $\begin{array}{c}\text { Grades of } \\
\text { Acne }\end{array}$ & $\begin{array}{c}\text { Low Serum Zinc } \\
\text { Levels } \\
(<\mathbf{o r}=\mathbf{7 0} \boldsymbol{\mu g} / \mathbf{d L})\end{array}$ & $\begin{array}{c}\text { Normal Serum } \\
\text { Zinc Levels }(>\mathbf{7 0} \\
\boldsymbol{\mu g} / \mathbf{d L})\end{array}$ & Total \\
\hline Group 1 & 7 & 51 & 58 \\
\hline Group 2 & 2 & 10 & 12 \\
\hline
\end{tabular}

Table 4. Serum Zinc Levels in Different Grades of Acne

\section{DISCUSSION}

\section{Age Incidence}

In a Swiss study, done by Bloch, it was found that 18 -year-old had the highest prevalence of clinical acne on the face- 35\% in men and $23 \%$ in women. It started earlier in boys $(10$ years) than in girls (11 years), whilst the maximum prevalence was later in girls (18 years) than the boys (17 
years). ${ }^{2}$

Hinrichsen et al and Hamilton et al found that acne started earlier and reached its maximum prevalence earlier in girls than in boys. The above studies had contradictory findings. ${ }^{2}$

In our study, the "maximum prevalence of acne was in the age group of 16 - 20 years (47.14\%). In boys, the maximum prevalence was at 18 years $(22.23 \%)$ and for girls it was at 18 and 19 years (both ages showed same prevalence of $17.65 \%$ ). This finding was in accordance with the studies done by Bloch as well as Thappa. ${ }^{3}$

Gotz et al observed that only $7 \%$ of cases developed acne before the age of 12 years and $40 \%$ experienced acne after they were 16 years. ${ }^{4}$ In the present study, $5.7 \%$ developed acne between 11 - 15 years of age and $94.3 \%$ of the patients developed acne after 16 years. Maximum cases had their age of onset between 16 - 20 years' age group. This finding coincides with the above finding. The reason for this finding is attributed to the increased androgen secretion with puberty.

The correct incidence and prevalence of acne could not be assessed with our study due to the small size of the study group. Most of the studies have been done in schools comprising of adolescents, whereas our study was not restricted to the adolescents only. Patients who attended the clinic with acne as their presenting complaint were included in the study.

\section{Family History}

Cunliff et al (1975) in one study found that $82 \%$ of the patients had a history of acne in at least one sibling and in $60 \%$ a history of acne was obtained from one or both the parents. ${ }^{5}$

In our study, $38.57 \%$ of patients had a family history of acne. $70.37 \%$ among these patients had a history of acne in at least one of the parents and remainder $29.63 \%$ of patients gave a history of acne in siblings. Heredity seems to have a role in the aetiology of acne, but it is not the only factor.

\section{Relation to Menstruation}

Cunliff et al (1974) demonstrated that acne got worse 5 - 6 days before the onset of menses. ${ }^{6}$ Cunliff and Cotterill (1976) observed that $60 \%-70 \%$ of the females notice a deterioration in their acne lesions in the premenstrual week. ${ }^{4}$ Vaswani and Pandhi (1991) found a significant decrease in the inflammatory and non-inflammatory lesions in the postmenstrual phase. Some studies report premenstrual exacerbation between $27 \%$ and $70 \%$.

In our study, $28(82.35 \%)$ of the females gave a history of premenstrual worsening of their acne lesions. This finding is in co-relation with all the above studies. The reason for this exacerbation has been attributed to hydration of the ductal pores premenstrually, causing increased resistance to sebum outflow. This leads to ductal obstruction and thus to the aggravation of lesions.

\section{Relation to Diet}

A controversy has long raged on the role of diet in the pathogenesis and management of acne. In a study by Rasmussen et al, $60 \%$ of the patients felt that chocolates and fatty foods were responsible for the flare-up of their acne lesions. Fulton et al did not find any effect of large amount of chocolates, milk, cold drinks and nuts on the acne lesions.
Vaswani and Pandhi did not find any relation between diet and the severity of lesions in their study. ${ }^{7}$ In 2001 , Green and Sinclair in their study showed the following results: $41 \%$ $(n=88)$ of medical students believed diet aggravated acne; $12 \%$ of these 88 blamed chocolate. 8

In the present study, $40 \%$ of the patients felt that fatty foods including fried and oily food stuffs (21.428\%), nonvegetarian food (14.285\%) and chocolates (4.285\%) were responsible for the flare-up of their acne lesions. This finding is in agreement with the Rasmussen study and Green and Sinclair study, but the reason for lower percentage could be a smaller size of the study group. Also, many of the patients were not in the habit of eating chocolates. Our findings were in contrast to those of Fulton et al and the reason for this is that their findings were based on the clinical trials and not on history as in our study.

\section{Type of Cosmetics Used}

Many of the cosmetics have comedogenic effect. There is a misconception among the acne patients that acne is caused by dirty skin. Thus, most of them end up washing their faces frequently with soaps and cleansers. In the process, they cause more harm to the already inflamed skin. Many of the patients have greasy skin and in spite of it may try to hide their lesions with harmful cosmetics. Moisturisers on a greasy skin will only serve to enhance the existing oiliness. In the present study $30 \%$ of the patients gave a history of using greasy cosmetics and $2.857 \%$ gave history of usage of nongreasy cosmetics. Besides 54 (77.14\%) of the patients described their skin type as oily. Thus, all these factors could have aggravated their acne.

\section{Seborrhoea}

One of the striking features of acne is a greasy skin, but all patients with seborrhoea do not have acne. Munro Asherman interviewed 490 subjects and found the incidence of dandruff to be $26 \%$ in patients with acne. ${ }^{4}$ In the present study, $54(77.14 \%)$ subjects with acne gave a history of seborrhoea. Our findings are not the same as the above studies, the reason being a small study group.

\section{Clinical Assessment of Acne}

Different grading methods have been used for measuring the severity of acne, but to date a widely accepted standardised classification system does not exist. A clinically meaningful objective assessment of Acne Vulgaris is very important when relating the relative merits of therapeutic substances in this condition. The assessment techniques usually employed are lesion counting, grading systems and photographic methods and each method has its positive and negative features.

Gibson et al (1984) compared different methods of evaluation including lesion counting, visual tactile grading and photography. They concluded that a well-thought-out clinical grading system is the best overall method, as it provides a meaningful assessment with speed and reasonable accuracy. ${ }^{9}$ Photography has a disadvantage that it cannot include the palpatory findings. Michaelsson evaluated their respective acne treatment results by counting all the evident lesions and multiplying with the respective severity index for each lesion. In the present study, 1 - 4 scale was used as described by Tutakne and Chari. It is an arbitrary method of grading acne severity, but a very quick and 
convenient one. Since the present study did not include any therapeutic trial, this method fulfilled the requirement of clinical assessment. This method helps in evaluating the patients and to choose the modality of treatment.

- Grade 1- Mainly comedones and occasional papules.

- Grade 2-Comedones, papules and occasional pustules.

- Grade 3- Mainly pustules and nodules.

- Grade 4- Nodules and Cysts.

In the present study, 18 patients $(25.714 \%)$ had grade I acne. 40 patients $(57.142 \%)$ had grade II acne. Most of the patients in this study belonged to grade II. $40 \%$ were females and $17.142 \%$ were males. $10(14.28 \%)$ had grade III and 2 (2.8\%) had grade IV acne and totally 12 patients $(17.142 \%)$ had severe acne. Among these, grade III and IV, 11 (15.714\%) were males and $1(1.428 \%)$ was female.

Our findings were different from the above studies, because their grading system was different and included only adolescents and also comprised of a large study group. Though the grading method used in the present study is similar to those used by many others, it has not been used in any study. It is just an arbitrary method of clinical assessment.

\section{Serum Zinc Levels}

The normal values of serum zinc levels appear to be rather uniform all over the world. Most laboratories have obtained a reference value of $70-125 \mu \mathrm{g} / \mathrm{dL}$.

Serum levels in acne patients have been studied by many workers with varying results. Michaelsson et al were the first to study zinc in relation to Acne Vulgaris. In their study (1977), they demonstrated a slight decrease in the serum zinc level in male patients with severe acne. Female patients did not show any definite change. They also found a definitive decrease in serum levels of retinol-binding difference in serum zinc levels between acne patients and healthy controls. Similarly, El-Saaiee et al could not demonstrate any change in zinc levels between acne patients and controls. In all the above studies, they demonstrated a beneficial effect of oral zinc in acne. Michaelsson (1990) studied both epidermal and serum zinc levels in acne and other skin disorders. He found low zinc levels in the epidermis, in spite of normal levels in the serum.

Amer et al (1982) demonstrated a significantly lower levels of serum zinc levels as compared to controls in both male and female acne patients with higher grades of acne.10 Their results were lower than those of Michaelsson et al. Women, whether diseased or healthy showed a significantly lower mean serum zinc levels as compared to men. Michaelsson et al, in contrast, did not show any decrease in the levels in females. Ghorpade et al (1982) studied serum zinc levels in 46 cases of Acne Vulgaris and 20 controls and found a significant decrease in the zinc levels in both males and females as compared to normal controls of same age. The levels were slightly lower in male patients, but this difference was not statistically significant. Madadi et al (1993) studied serum zinc levels and other trace elements in acne patients and found significant decrease in the serum zinc levels as compared to those of controls. They however did not study the differences in male and female patients. ${ }^{3}$
In the present study, the serum zinc levels were studied in 70 acne patients. Serum zinc levels were not significantly low in acne patients of different grades of the same age group, since probability value $P>0.05$. It is not statistically significant at $5 \%$ level of significance. Therefore, there is no correlation between serum zinc levels and different grades of acne.

Our findings were similar to Michaelsson et al (1978) and El-Saaiee studies. Few workers have found zinc levels to be significantly low only in males with severe acne and others have found it decreased in both male and female acne patients irrespective of their acne score and yet others have found no change in the serum zinc levels between acne patients and normal controls.

\section{CONCLUSION}

Among the 70 cases studied, maximum cases were seen in the 16 - 20 years' age group (47.14\%). Male-to-female ratio was 1.0588: 1. A significant family history was noted. Summer and premenstrual exacerbations were observed. Seborrhoea was an important associated feature. A significant number of patients blamed diet for aggravation of their lesions. Most cases belonged to grade II acne. Males had more severe acne than females.

Serum zinc levels were not significantly low in acne patients of different grades of the same age group. There was no correlation between the severity of acne and serum zinc levels.

\section{REFERENCES}

[1] Koo J. The psychological impact of acne: patients' perceptions. J Am Acad of Dermatol 1995;32(5 Pt 3):S26-S30.

[2] Burton IL, Cunliff WJ, Stafford I, et al. The prevalence of acne vulgaris in adolescence. $\mathrm{Br} \mathrm{J}$ Dermatol 1971;85(2):119-26.

[3] Schafer T, Nienhaus A, Vieluf D, et al. Epidemiology of acne in the general population: the risk of smoking. British Journal of Dermatology 2001;145(1):100-4.

[4] Cunliff WJ, Cotterill JA. The acnes, cinical features, pathogenesis and treatment. London: WB Saunders 1975.

[5] Walton S, Wyatt EH, Cunliffe WJ. Genetic control of sebum excretion and acne- a twin study. Br J Dermatol 1988;118(3):393-6.

[6] Cunliff WJ. Acne. $1^{\text {st }}$ edn. London: Martin Dunitz 1989.

[7] Vaswani NK, Pandhi RK. Acne vulgaris and diet. Ind J Dermatol Venereol Leprol 1991;57:138-40.

[8] Spencer EH, Ferdowsian HR, Barnard ND. Diet and acne: a review of the evidence. International Journal of Dermatology 2009;48(4):339-47.

[9] Gibson JR, Harvey GS, Barth J, et al. Assessing inflammatory acne vulgaris-correlation between clinical and photographic methods. Br J Dermatol 1984;111(Suppl 27):168-70.

[10] Amer M, Bhagat MR, Tosson Z, et al. Serum zinc in acne vulgaris. Int J Dermatol 1982;21(8):481-4. 